\title{
cAMP elevators inhibit LPS-induced IL-12 p40 expression by inter- fering with phosphorylation of p38 MAPK in Murine Peritoneal Macrophages
}

\author{
Wei Guo FEnG, Yi Bing WANG, Jin Song ZHANG, Xing Yu WANG, Chang lin LI, Zong \\ LIANG CHANG* \\ Laboratory of Immune Signaling Transduction, Institute of Biochemistry and Cell Biology, Shanghai Institutes for \\ Biological Sciences, Chinese Academy of Sciences, Shanghai 200031, China
}

\begin{abstract}
mediated signaling may play a suppressive role in immune response. We previously found that the cAMPelevators (CTx and 8-Br-cAMP) inhibited IL-12, IL-1a, IL-6 gene expression, but increased the transcriptional levels of IL-10 and IL-1Ra in LPS-treated murine peritoneal macrophages. The present study examined a possible molecular mechanism involved in cAMP elevators-induced inhibition of IL-12 p40 expression in response to LPS. Our data demonstrated that cAMP elevators downregulated IL-12 p40 mRNA expression and IL-12 p70 production in murine peritoneal macrophages. Subsequent studies revealed that cAMP-elevators blocked phosphorylation of p38 MAPK, but did not affect the activity of NF- $\kappa$ B binding to IL-12 promoter (-136/-112). This is the first report that cAMP elevators inhibit LPS-induced IL-12 production by a mechanism that is associated, at least in part, with p38-dependent inhibition by cAMP signaling pathways.
\end{abstract}

Key words: $I L-12, c A M P, L P S, N F-\kappa B, p 38 M A P K$.

\section{INTRODUCTION}

By releasing different kinds of cytokines, macrophages play a central role in the inflammatory reaction, as well as determining, initiating and maintaining the specific immune response. In response to microbes and their products, such as LPS (lipopolysaccharides), macrophages can secret a number of pro-inflammatory cytokines, including IL-12, IL-1, IL-6 and TNF- $\alpha$ [1-5]. They can also produce a number of anti-inflammatory cytokines, such as TGF- $\beta$, IL-10 and IL-1R $\alpha[6-8]$. The balance of the pro-inflammatory cytokines and anti-inflammatory cytokines determines the consequent immune response.

* Corresponding author: Prof. Zong Liang CHANG Fax: 0086-21-64331090 E-mail: immusig@sunm.shenc.ac.cn Abbreviations: IL-12, interleukin 12; NF- $\kappa$ B, nuclear factor $\mathrm{kB}, \mathrm{TNF}-a$ trmor necrosis factor- $a$
The balance of pro-inflammatory cytokines and anti-inflammatory cytokines is mainly controlled by the characteristics of pathogens. However, it can also be regulated by exogenous drugs. cAMP-inducing drugs were widely used to suppress inflammation, as well as cellular immune responses[9],[10]. In our previous experiments cAMP-inducers such as CTx (cholera toxin) and 8-Bromo-cAMP (8-Br-cAMP, a cellular permeable cAMP) were found to decrease the production of pro-inflammatory cytokines, including TNF- $\alpha$, IL-6, IL-12, and increase the secretion of anti-inflammatory cytokines, such as IL-10 and IL-1Ra[1],[11]. Other cAMP inducers like PACAP (pituitary adenylate cyclase-activating polypeptide) and VIP (vasoactive intestinal peptide) have been reported to show the similar effect on cytokines expression by macrophages[12],[13].

IL-12 provides an important link between innate 
immunity and adaptive immunity, favoring the development of $\mathrm{CD} 4^{+}$Th1 cell-mediated immune response that can better protect the host against viruses and bacteria[14]. The pivotal role of IL-12 in both innate immunity and adaptive immunity generated the interest of regulation of its gene transcription. IL-12 is a disulfide-linked heterodimer of $35 \mathrm{KD}$ (p35) and 40KD (p40) subunits. Although these subunits share regions of homology, they also have unique structural features and their gene expression regulation is distinct. IL-12 p35 subunit is synthesized in many cells and tissues, while p40 synthesis is largely restricted to activated mononuclear phagocytes and dendritic cells[14],[15]. As a result, IL-12 p40 subunit expression is often considered to be the limiting factor for IL-12 expression. In present study, we focused our research on IL-12 p40 expression.

There are many reports that LPS can greatly augment IL-12 expression in macrophages[16-18]. In our previous experiments, we have demonstrated that both p38 MAPK (mitogen-activated protein kinase) and NF-kB are same important to LPS-induced IL12 gene expression[16]. In addition, NF-kB binding activity with IL-12 promoter (-112bp, -136bp) is independent of the p38 MAPK because SB203580 (a specific inhibitor of P38 MAPK signaling pathway) can decrease LPS-induced IL-12 expression without affecting the binding activity of NF-kB binding to IL-12 p40 promoter region[16].

It has also been shown by other labs and us that LPS-induced IL-12 expression can be greatly downregulated by cAMP inducers[1],[13],[19], [20]. However there is little information about the mechanism for cAMP-signal-inhibitory effect on LPS-induced IL-12 expression in macrophages. There is a single report on this subject in macrophage cell line [15]. The mechanism in murine or human macrophages is still unclear. We hypothesized that cAMPsignal may inhibit IL-12 expression through inhibiting the p38 MAPK, or NF- $\kappa$ B binding activity. In the present study, we examine this hypothesis in murine peritoneal macrophages.

\section{MATERIALS AND METHODS}

\section{Preparation of murine peritoneal macrophages}

Peritoneal macrophages were harvested from two-month-age male C57BL/6J mice that were injected ip $4 \mathrm{~d}$ earlier with $2 \mathrm{ml}$ of
$5 \%$ thioglycollate medium (Sigma). Cells were seeded in 6-cm plates and cultured at $37{ }^{\circ} \mathrm{C}$ in a humidified atmosphere containing $5 \%$ $\mathrm{CO}_{2}$ with RPMI 1640 medium (GIBCO) including $10 \mathrm{~m} M$ Hepes buffer, $100 \mathrm{U} / \mathrm{ml}$ penicillin and $100 \mu \mathrm{g} / \mathrm{ml}$ streptomycin. After incubation for $4 \mathrm{~h}$, nonadherent cells were removed, and the adherent cells were cultured and stimulated for different periods of time in phenol red-free RPMI 1640 medium.

\section{Western blot}

Treated peritoneal macrophages were lysed in SDS-sample buffer [62.5 m M Tris-HCl (pH6.8), 2\% w/v SDS, 10\% glycerol, 50 $\mathrm{mM}$ DTT, and $0.1 \% \mathrm{w} / \mathrm{v}$ bromophenol blue] and the cellular DNA was sheared by sonication. Proteins were analyzed in 10\% SDSPAGE and transferred to a nitrocellulose membranes (Schleicher and Schuell). Membranes were incubated overnight at $4{ }^{\circ} \mathrm{C}$ with primary antibody (1:1000 dilution, anti-phosphorylated p38 Ab, from New England Biolabs) followed by a horseradish peroxidase conjugated secondary antibody (1:1000 dilution) for $1.5 \mathrm{~h}$ at room temperature. The signals were visualized by an enhanced chemiluminescence system (ECL) according to the manufacturer' $\mathrm{s}$ recommendation (New England Biolabs, MA).

\section{Nuclear extraction}

The nuclear extraction procedure was described in[16].

\section{Electrophoretic mobility shift assays (EMSAs)}

Oligonucleotide (5'-CTT AAA ATT CCC CCA GAA TGT TTT G $-3^{\prime}$ ) with sequence corresponding to NF- $\kappa$ B binding site (underline) of the murine IL-12 p40 promoter (-136/-112) was synthesized and annealed with its complementary sequence by incubation at $90^{\circ} \mathrm{C}$ for $10 \mathrm{~min}, 65^{\circ} \mathrm{C}$ for $10 \mathrm{~min}, 37^{\circ} \mathrm{C}$ for $30 \mathrm{~min}$ in $10 \mathrm{~m} M$ Tris- $\mathrm{HCl}, 50 \mathrm{mM} \mathrm{NaCl}, 10 \mathrm{~m} \mathrm{M} \mathrm{MgCl}$, and $1 \mathrm{mM}$ DTT. The NF-kB consensus oligonucleotide (5'-AGT TGA GGG GAC TTT CCC AGG C-3') and CREB consensus oligonucleotide were purchased from Santa Cruz Biotechnology, Inc, California. The oligonucleotide probes were end-labeled using $\left[\gamma-{ }^{32} \mathrm{P}\right]$ ATP (Amersham Pharmacia Biotech) and T4-polynucleotide kinase (Promega). The nuclear protein-DNA binding reaction was carried out at room temperature for $20 \mathrm{~min}$ in a volume of $20 \mu \mathrm{l}$ containing $10 \mu \mathrm{g}$ nuclear extract protein, $10 \mathrm{mM}$ Tris-HCl (pH7. 5), $50 \mathrm{mM} \mathrm{NaCl}, 1 \mu \mathrm{M}$ EDTA, $5 \%$ glycerol, $1 \mathrm{mM}$ DTT and $1 \mathrm{mg}$ of poly (dI-dC) as a non-specific competitor. The DNA-protein complexes were resolved by electrophoresis through $6 \%$ polyacrylamide gels containing $50 \mathrm{~m} M$ Tris, $0.38 M$ glycine and $2 \mathrm{~m} M$ EDTA. The gels were dried and autoradiographed with intensifying screens at $-70{ }^{\circ} \mathrm{C}$. Supershift assays were performed as described above with the exception that subsequent to incubation of oligonucleotide probes with nuclear extracts, $2.0 \mu \mathrm{g}$ of antibodies (antip50, p52, p65, c-Rel, Rel B from Santa Cruz Biotechnology, Inc, California.) were added to the reaction mixture respectively and incubated for $30 \mathrm{~min}$ at room temperature.

RNA isolation and ribonuclease protection assay (RPA)

After treatment, peritoneal macrophages were homogenized with TRIzol (GIBCOL BRL). RNA was separated with chloroform, 
precipitated with isopropyl alcohol, washed with $75 \%$ ethanol, and redissolved in the water treated with $0.1 \%$ DEPC (diethylpyrocarbonate, RNase inhibitor). RPA kit (Pharmingen company) was used to quantify several cytokines' mRNA at the same time. After hybridization with the $\left[\alpha-{ }^{32} \mathrm{P}\right] \mathrm{UTP}$ labeled antiRNA probes of many cytokines, the total RNA samples were digested by $\mathrm{S} 1 \mathrm{RNase}$ which can only hydrolyze the single-chain RNA. Then the labeled double-chain RNA was purified, denatured and separated in 5\% PAGE gel with 8M Urea. Finally, the gel was dried, exposed and quantitated in a phosphorImager (Moleculor Dynamics, Sunnyrale, CA) by using the ImageQuant software. Every gene has a corresponding band on the film, and the OD of the band shows the quantity of the corresponding mRNA.

\section{ELISA for IL-12 p70}

After treatment, culture supernatants were collected and the IL-12 protein was determined by using an ELISA kit (Quantikine mouse IL-12 p70 Immunoassay, R \& D Systems, Inc., Minneapolis, $\mathrm{MN}$ ) according to the manufacturer' $\mathrm{s}$ instructions.

\section{RESULTS}

\section{Effect of cAMP-signal on IL-12 mRNA expression} and protein secretion

In order to confirm the relationship between the IL-12 p40 mRNA expression and cAMP-signal, doseresponses of 8-Br-cAMP and CTx were studied. In Fig 1, our RPA results clearly showed the inhibitory

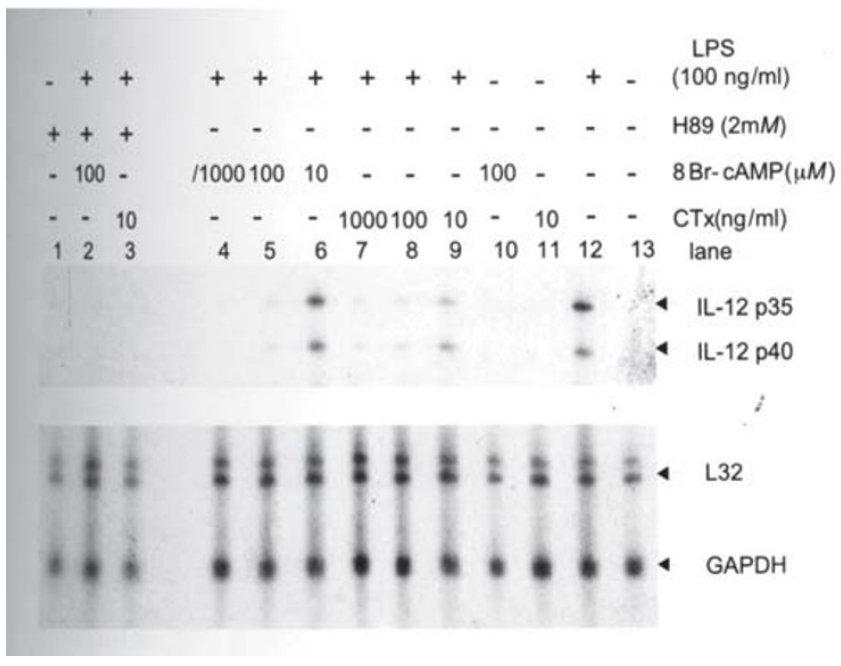

Fig 1. 8-Br-cAMP and CTx inhibit LPS-induced IL-12 mRNA expression and the inhibitory effect is in a dose- dependent manner. This result was obtained by RPA. L32 and GAPDH were all housekeeping genes whose expression was detected at the same time as IL-12 p35 and p40. After pretreated by 8-Br-cAMP, CTx or H89 or none for 15 mins, peritoneal macrophages were stimulated by LPS for $4 \mathrm{hrs}$ before mRNA was extracted for RPA. effect of cAMP-signals on LPS-induced IL-12 p40 subunit expression, and this inhibitory effect was in a dose-dependent manner.

At the same time, the relationship between the IL-12 p70 protein secretion and cAMP signal was also investigated. In Fig 2, ELISA results indicate that cAMP signal can inhibit LPS-induced IL-12 p70 protein secretion as well. It is worth pointing out that, the inhibitory effect of cAMP signal on IL-12 gene expression does not depend on the activity of PKA (protein kinase A). In our experiments, H89, one of specific inhibitor of PKA, reversed inhibition of cAMP-signal at a very limited level, even when H89 was at high concentration of $1 \mu M$ or $2 \mu M$ (H89 $\mathrm{Ki}$ for PKA is $48 \mathrm{nM}$ ).

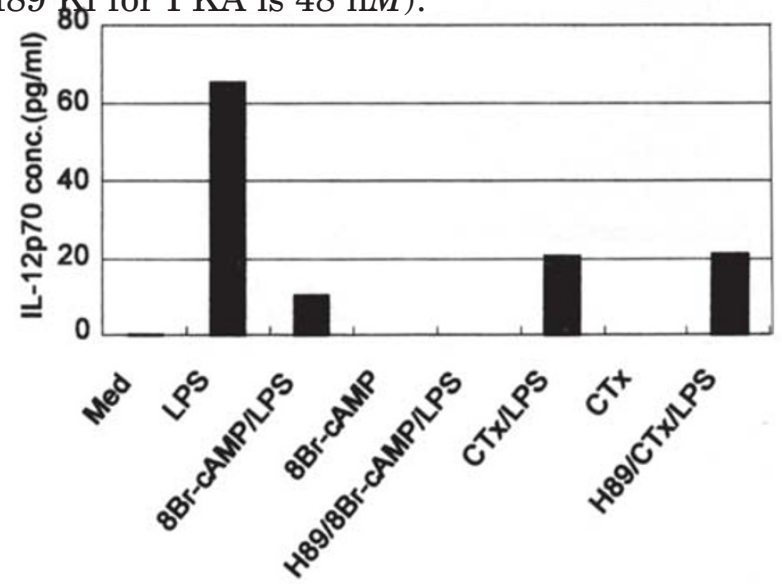

Fig 2. $8 \mathrm{Br}$-cAMP and CTx can inhibit LPS-induced IL-12 p70 production, and the inhibitory effect can not be restored by H89. With or without H89 $(2 \mathrm{mM})$ pretreatment for $30 \mathrm{~min}$, peritoneal macrophages were first treated by 8 -Br-cAMP (100 $\mu M)$ or CTx $(100 \mathrm{ng} / \mathrm{ml})$ or none for another $30 \mathrm{~min}$, then stimulated by LPS (100 ng/ml) for $24 \mathrm{~h}$. The supernatants of peritoneal macrophages were collected and IL-12p70 concentration was measured and standardized by using Quantikine mouse IL-12 p70 ELISA kit. Three independent experiments were performed and one of typical results was shown here.

\section{$N F-k B$ and $I L-12$ p40 promoter region}

In contrast with untreated peritoneal macrophages, LPS can greatly increase the NF- $\kappa \mathrm{B}$ binding to the (-136/-112) region of the IL-12 p40 promoter, and two binding bands were shown in Fig 3. In order to identify these two binding bands, we used antibodies (Ab) specific for NF- $\kappa$ B family members. As seen in Fig 3, p50 Ab can supershift both protein bands, p65 Ab supershifts only the last 
protein bands whereas the other Abs (p52, c-Rel, Rel-B Abs) did not supershift any protein band. So, the leading protein band was the homodimer of $\mathrm{p} 50$ / $\mathrm{p} 50$, and the other was the heterodimer of $\mathrm{p} 50 / \mathrm{p} 65$. Both binding bands can be positively regulated by LPS.

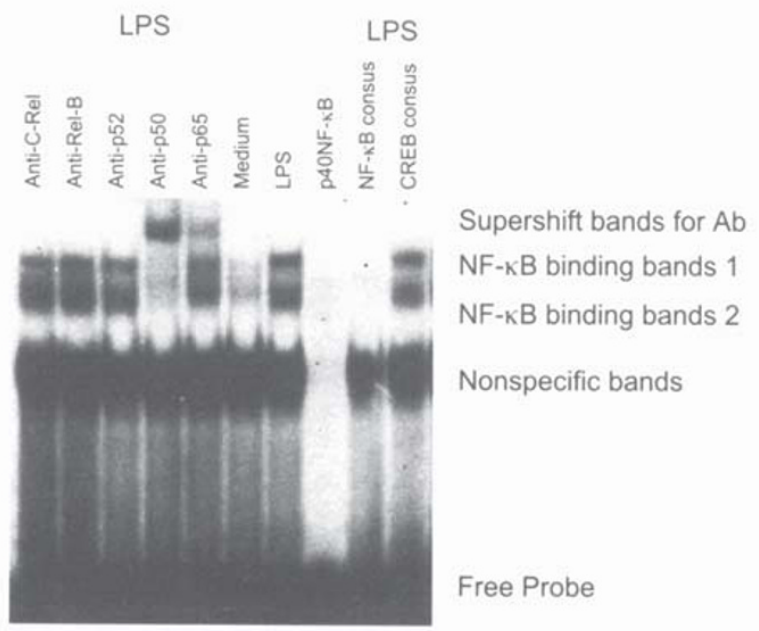

Fig 3. Identify the complexes binding to IL-12 p40 NF- $\kappa$ B site (-136/-112) by EMSA. After $1 \mathrm{~h}$ treatment of LPS or none, peritoneal macrophages were lysed and nuclear proteins were prepared according to the procedure. Nuclear protein concentration was measured and aliquoted into $10 \mathrm{mg}$ for each EMSA reaction. In supershift or competition experiment, $1 \mathrm{ml}$ corresponding antibody or $50 \mathrm{x}$ cold dsDNA oligonucleutides was added to nuclear protein and incubated on ice for half an hour before EMSA procedure was performed.

In an effort to further demonstrate the specificity of NF- $\kappa$ B binding to IL-12 p40 promoter, competition experiments were carried out. As shown in Fig 3 , only unlabelled IL-12 p40 promoter and NF- $\kappa$ B consensus sequence could compete for the binding of $\mathrm{NF} \kappa \mathrm{B}$ protein to IL-12 promoter, while CREB consensus did not, even at a concentration 100 times higher than that of ${ }^{32} \mathrm{P}$-labelled probe.

cAMP-signal and NF- $\kappa B$ binding activity with Il12 p 40 promoter

Activity of members of NF-kB family is the hallmark of macrophage activation by bacterial products and pro-inflammatory cytokines. Moreover NF- $\kappa$ B binding to IL-12 p40 promoter is essential for LPSinduced IL-12 p40 expression[16].

In order to study whether cAMP-signal affects LPS-induced NF- $\kappa$ B binding activity with IL-12 p40 promoter, different concentration of 8-Br-cAMP and CTx were used. In Fig 4, LPS-induced NF- $\kappa$ B ac- tivity was not inhibited by cAMP-inducers and did not show a dose-dependent inhibitory effect by $8 \mathrm{Br}$ cAMP and CTx. That is the inhibitory effect of 8Br-cAMP and CTx on LPS-induced IL-12 p40 expression is independent of NF- $\kappa \mathrm{B}$ binding activity.

In Fig 4, SB203580 (4-(4-Fluorophenyl)-2-(4methylsulfinylphenyl)-5-(4-pyridyl) $1 \mathrm{H}$-imidazole, a specific inhibitor for p38 MAPK) also did not show any inhibitory effect on LPS-induced NF- $\kappa$ B binding activity although different doses were investigated.

\section{cAMP signal and LPS-induced p38 MAPK activity}

p38 MAPK is activated only when its Tyr182 is phosphorylated. Through western blotting, westudied the relationship between cAMP signal and p38 MAPK phosphorylation (Fig 5). LPS treatment stimulates phosphorylation of p38 MAPK in murine peritoneal macrophages. Moreover the activity of p38 has been previously proved to be essential for maximal expression of IL-12 p40 in LPS-stimulated peritoneal macrophages. Pretreatment of macrophages with CTx or 8-Br-cAMP inhibited LPS-induced p38 MAPK phosphorylation in a dose-dependent manner.

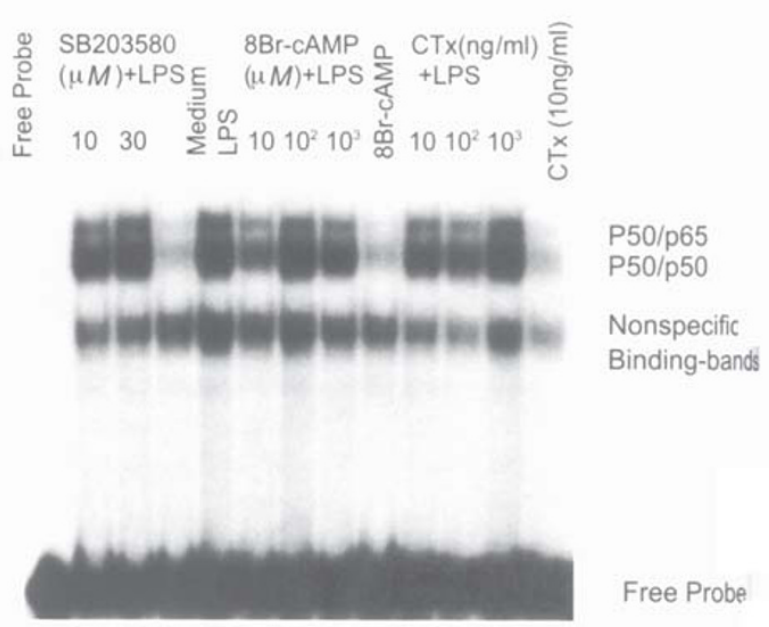

Fig 4. 8-Br-cAMP and CTx do not show dose-dependent inhibitory effect on LPS-induced NF- $\kappa$ B binding to IL-12 p40 Promoter. After pretreated by 8-Br-cAMP, CTx or SB203580 for $30 \mathrm{~min}$, peritoneal macrophages were then treated by LPS for another $3 \mathrm{~h}$. The EMSA was performed by using the nuclear extract from these cells. 


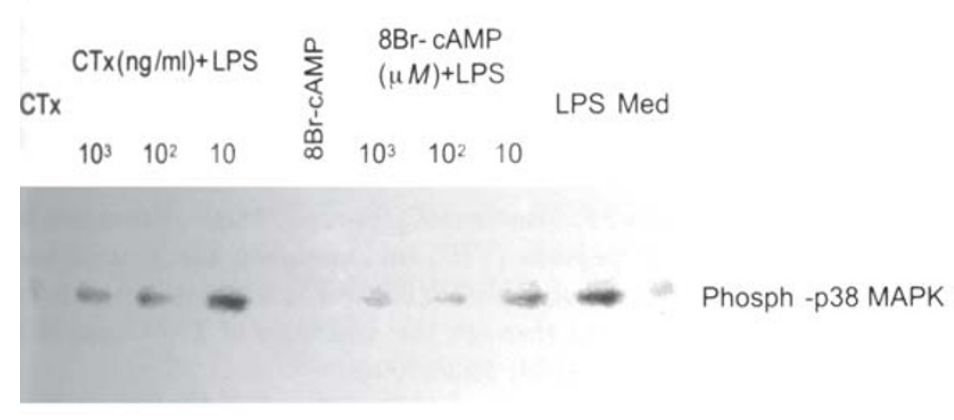

Fig 5. 8-Br-cAMP and CTx can inhibit LPS-induced p38 MAPK phosphorylation, and the inhibitory effect is in a dose-dependent manner. Before $3 \mathrm{~h}$ LPS treatment, peritoneal macrophages were pretreated with 8-Br-cAMP or CTx for $30 \mathrm{~min}$. The total protein was extracted for western blot. This is one representative result of three repeated experiments.

\section{DISCUSSION}

The present study provides partial support for our hypothesis that 8-Br-cAMP and CTx mediated the inhibition of p38 MAPK activity was associated with downregulation of IL-12 mRNA expression and IL-12 p70 secretion. Although it is not clear which substrate of p38 MAPK mainly contributes to IL12 p40 expression, inhibition of p38 MAPK certainly decreased IL-12 p40 expression according to previous report[16]. ATF-2, a possible substrate for p38 MAPK[22-24], was also investigated. 8-BrcAMP and CTx inhibited LPS-induced ATF-2 activity and the effect was in a dose-dependent manner similar to the results with p38 MAPK (data not shown). It is possible that ATF-2 was the transcription factor that controlled LPS-induced IL-12 p40 expression. However, untill now an ATF-2 binding site on IL-12 promoter has not been described. Additionally, other downstream transcriptional factors of p38 MAPK, such as ATF-1 and CREB (cAMP response element binding protein)[25], may play critical roles on IL-12 expression. However, till now, no functional CRE (cAMP response element), which is able to bind with CREB and ATF-1 proteins, has been found in IL-12 p40 promoter region. Therefore, further investigation of the IL-12 p40 promoter region is needed. In general, we are not certain whether ATF-2 is essential for inhibitory effect of 8-Br-cAMP and CTx on LPS-induced IL12 p40 expression. However we demonstrated that inhibition of p38 MAPK activity can explain, at least in part, the inhibitor effect of $8-\mathrm{Br}$ cAMP and CTx.

We have also examined the effect of cAMPelevator on MAPKs other than p38 MAPK. Our published data[26] showed that cAMPelevators were able to inhibit LPS-induced ERK1/2, JNK1/2 phosphorylation, but p38 MAPK appeared to be only associated with IL-12 expression[16].

Surprisingly, 8-Br-cAMP and CTx did not affect LPS-induced NF- $\kappa$ B binding to IL-12 p40 promoter. These results differ from results obtained with the macrophages cell line Raw 264.7. Mario and his colleagues showed an inhibitory effect of PACAP or LPS-induced NF- $\kappa$ B binding to IL-12 p40 promoter in Raw 264.7[13],[15]. These differences may be due to different experimental systems. To simplify the experimental design and focus on the mechanism of inhibitory effect of cAMP-elevators on IL-12 p40 expression, we did not use IFN- $\gamma$ to prime peritoneal macrophages like Mario. Although IFN- $\gamma$ can synergistically increase LPS-induced IL-12 p40 expression and make IL-12 p40 mRNA easier to detect[16],[27], IFN- $\gamma$ itself can induce other complex reactions in LPS-treated macrophages.

IL-12 is one of the pro-inflammatory cytokines that are mainly secreted by activated macrophage. IL-12 can transform the activation of macrophage into the development and activation of $\mathrm{CD} 4^{+} \mathrm{Th} 1$ cells, lead innate immune response to cell-mediated immune response, and magnify the primary inflammatory reaction[9],[28],[29]. Although inflammation is a protective activity, however, under certain conditions such as severe infection, excessive cytokines could cause the collapse of the immune system. Therefore, it is important to find the way that over expression pro-inflammatory cytokine, such as IL12 can be downregulated. Currently, our data provided a possible mechanism for 8-Br-cAMP and CTx to suppress LPS-induced IL-12 p40, which provide us the basis to develop new immunomodulators for therapeutic application. Since cAMP-elevator can bring about multiple effects on macrophages, our study make it possible to find new substitute drugs for cAMP-elevator, which can inhibit IL-12 expression without affecting other cytokine genes 
expression.

\section{ACKNOWLEDGEMENTS}

This work was supported by Shanghai Institutes for Biological Sciences, Chinese Academy of Sciences, and the grant from Ministry of Science and Technology (G1999054201) and National Natural Science Foundation of China (39870717). We would like to thank Prof. Kozel TR of Department of Microbiology of University Nevada, Reno, USA for his helpful reviewing of the manuscript.

\section{REFERENCES}

[1] Feng W, Wang Y, Zhang J, Wang X, Li C, Chang Z. Effects of CTx and 8-bromo-cAMP on LPS-induced gene expression of cytokines in murine peritoneal macrophages. Biochemical and Biophysical Research Communications 2000; 269:570-3.

[2] Caivano M, Cohen P. Role of mitogen-activated protein kinase cascades in mediating lipopolysaccharide-stimulated induction of cyclooxygenase- 2 and IL- 1 beta in RAW264 macrophages. J Immunol 2000; 164:3018-25.

[3] Remick DG, Newcomb DE, Bolgos GL, Call DR. Comparison of the mortality and inflammatory response of two models of sepsis: lipopolysaccharide vs. cecal ligation and puncture. Shock 2000; 13:110-6.

[4] Cambronero R, Sewell WA, North ME, Webster AD, Farrant J. Up-regulation of IL-12 in monocytes: a fundamental defect in common variable immunodeficiency. J Immunol 2000; 164:488-94.

[5] Nick JA, Young SK, Brown KK, et al. Role of p38 mitogenactivated protein kinase in a murine model of pulmonary inflammation. J Immunol 2000; 164:2151-9.

[6] Kobayashi H, Kobayashi M, Heming TA, Bidani A, Pollard RB, Suzuki F. Cytokine production by rabbit alveolar macrophages: differences between activated and suppressor cell phenotypes. Immunol Lett 1999; 69:339-46.

[7] Brightbill HD, Plevy SE, Modlin RL, Smale ST. A prominent role for Sp1 during lipopolysaccharide-mediated induction of the IL-10 promoter in macrophages. J Immunol 2000; 164:1940-51.

[8] Pue CA, Mortensen RF, Marsh CB, Pope HA, Wewers MD. Acute phase levels of C-reactive protein enhance IL-1 beta and IL-1ra production by human blood monocytes but inhibit IL-1 beta and IL-1ra production by alveolar macrophages. J Immunol 1996; 156:1594-600.

[9] Kammer GM. The adenylate cyclase-cAMP-protein kinase A pathway and regulation of the immune response. Immunol Today 1988; 9:222-9.

[10] Uotila P. The role of cyclic AMP and oxygen intermediates in the inhibition of cellular immunity in cancer. Cancer Immunol Immunother. 1996; 43:1-9.

[11] Zhong WW, Burke PA, Drotar ME, Chavali SR, Forse RA. Effects of prostaglandin E2, cholera toxin and 8-bromocyclic AMP on lipopolysaccharide-induced gene expression of cytokines in human macrophages. Immunology
1995; 84:446-52.

[12] Goetzl EJ, Pankhaniya RR, Gaufo GO, Mu Y, Xia M, Sreedharan SP. Selectivity of effects of vasoactive intestinal peptide on macrophages and lymphocytes in compartmental immune responses. Ann N Y Acad Sci 1998; 840:540-50.

[13] Delgado M, Martinez C, Pozo D, et al. Vasoactive intestinal peptide (VIP) and pituitary adenylate cyclase-activation polypeptide (PACAP) protect mice from lethal endotoxemia through the inhibition of TNF-a and IL-6. J Immunol 1999; 162:1200-5.

[14] Abbas AK, Lichtman AH, and Pober JS. Eds. Cellular and Molecular Immunology. Saunders Press: Philadelphia 1997:249-77.

[15] Delgado M, Ganea D. Vasoactive intestinal peptide and pituitary adenylate cyclase-activating polypeptide inhibit interleukin-12 transcription by regulating nuclear factor $\mathrm{kB}$ and Ets activation. J Biol Chem 1999; 274: 31930-40.

[16] Zhang JS, Feng WG, Li CL, Wang XY, Chang ZL. NF-kB regulates the LPS-induced expression of interleukin 12 p40 in murine peritoneal macrophages: roles of PKC, PKA, ERK, p38 MAPK, and proteasome. Cell Immunol 2000; 204:38-45.

[17] Lu HT, Yang DD, Wysk M, Gatti E, Mellman I, Davis RJ, Flavell RA. Defective IL-12 production in mitogen-activated protein (MAP) kinase kinase 3 (Mkk3)-deficient mice. EMBO J 1999; 18:1845-57

[18] Aicher A, Shu GL, Magaletti D, Mulvania T, Pezzutto A, Craxton A, Clark EA. Differential role for p38 mitogenactivated protein kinase in regulating CD40-induced gene expression in dendritic cells and B cells. J Immunol 1999; 163:5786-95.

[19] Szabo G, Girouard L, Mandrekar P, Catalano D. Regulation of monocyte IL-12 production: augmentation by lymphocyte contact and acute ethanol treatment, inhibition by elevated intracellular camp. Int J Immunopharmacol 1998; 20:491-503.

[20] Link AA, Kino T, Worth JA, McGuire JL, etal. Ligandactivation of the adenosine A2a receptors inhibits IL-12 production by human monocytes. J Immunol 2000; 164: 436-42.

[21] Andrews NC, Faller DV. A rapid micropreparation technique for extraction of DNA-binding proteins from limiting numbers of mammalian cells. Nucleic Acids Res 1991; 19:2499.

[22] Zhu T, Lobie PE. Janus kinase 2-dependent activation of p38 mitogen-activated protein kinase by growth hormone. Resultant transcriptional activation of ATF-2 and CHOP, cytoskeletal re-organization and mitogenesis. J Biol Chem 2000; 275:2103-14.

[23] Brinkman BM, Telliez JB, Schievella AR, Lin LL, Goldfeld AE. Engagement of tumor necrosis factor (TNF) receptor 1 leads to ATF-2- and p38 mitogen-activated protein kinase-dependent TNF-alpha gene expression. J Biol Chem 1999; 274:30882-6.

[24] Hanafusa H, Ninomiya-Tsuji J, Masuyama N, et al. Involvement of the p38 mitogen-activated protein kinase pathway in transforming growth factor-beta-induced gene 
expression. J Biol Chem 1999; 274:27161-7.

[25] Guha M, Mackman N. LPS induction of gene expression in human monocytes. Cell Signal 2001; 13:85-94.

[26] Lin MQ, Chang ZL. Immunomodulated Signaling in Macrophages: Regulation of the MAPK Signaling Pathways by PKA and PKC. Acta Biochimica et Biophysica Sinica 1999; 31:701-6.

[27] Feng WG, Chang ZL. Expression of cytokine mRNA during immuno-modulation of murine suppressor macrophages. Cell Res 1998; 8:317-22.

[28] Hu YJ, Zang L, Wu YD, Sun B. High IFN-a expression is associated with the induction of experimental autoimmune uveitis (EAU) in Fischer 344 rat. Cell Res 2001; 11:293-300.

[29] Geng JG. Directional migration of leukocytes: their pathological roles in inflammation and strategies for development of anti-inflammatory therapies. Cell Res 2001; 11:85-8. 